\title{
Nasal polyps do not contain olfactory structures*
}

\author{
Mahmood F. Bhutta ${ }^{1}$, Salim Al-Shaikh², Mo Latif ${ }^{2}$, Robin Lee², Jamal Uraiby ${ }^{3}$ \\ Nuffield Department of Surgical Sciences, University of Oxford, United Kingdom \\ Department of ENT, Kettering General Hospital, Kettering, United Kingdom \\ 3 Department of Pathology, Kettering General Hospital, Kettering, United Kingdom
}

\begin{abstract}
SUMMARY
Background: Nasal polyposis can lead to olfactory dysfunction, either due to physical obstruction of the olfactory cleft or physiological disruption of the olfactory neuroepithelium. Where medical therapy has failed to relieve symptoms of nasal polyposis, surgical excision can be considered. However, removal of polyps medial to the middle turbinate is controversial: some believe this will relieve physical obstruction to odourants, others state that removal here risks excising olfactory neuroepithelium.

Methods: We stained 25 nasal polypectomy samples from the area medial to the middle turbinate with olfactory marker protein.

Results: We confirmed that our staining method worked on normal olfactory tissue. However, no positive staining of nasal polyps was demonstrated.

Conclusion: We conclude that nasal polyps medial to the middle turbinate do not contain olfactory neurons, and surgical excision is not contraindicated.
\end{abstract}

Key words: nasal polyps, surgery, smell, olfactory mucosa

\section{INTRODUCTION}

Nasal polyposis can lead to nasal obstruction as well as hyposmia. Surgical polypectomy can be used for symptoms of nasal obstruction where medical therapy has failed, but the role of surgery for symptoms of hyposmia is more controversial. In some cases, polypectomy appears to improve olfactory function, whereas in other cases it makes little difference.

One key question here is whether nasal polyps arising near to the olfactory cleft may themselves contain olfactory components. If they do, obviously they should not be excised if the aim is for olfactory function to be restored. In contrast, if they do not, there may be an argument that, empirically, removal of these polyps may be beneficial in allowing odourants to reach the olfactory neuroepithelium.

Olfactory marker protein (OMP) is a $19-\mathrm{kDa}$ protein found abundantly and specifically in mature olfactory neurons. It is believed to play a role in signal transduction and axonal guidance ${ }^{(1)}$. Using anti-OMP antibodies, we demonstrate in this study that inflammatory nasal polyps do not contain olfactory neurons.

\section{MATERIALS AND METHODS}

Optimisation and confirmation of staining method

Whereas olfactory marker protein is phylogenetically conserved, most mammalian studies on its function have been undertaken in rodents, with only a few human studies ${ }^{(2-4)}$. We first optimized histological processing by trialing our staining protocol on a sample of normal olfactory epithelium. This sample was excised from the anterior superior turbinate of a lady, shortly after she died of metastatic colonic carcinoma. This lady did not suffer from nasal polyposis.

The excised tissue was processed for light microscopy and immunohistochemistry. For light microscopic examination, tissue was fixed in $10 \%$ neutral-buffered formalin, processed and embedded in paraffin wax. Tissue blocks were sectioned at $3 \mu \mathrm{m}$ and slides stained with haematoxylin and eosin. For immunohistochemistry, $3 \mu \mathrm{m}$ formalin fixed sections were prepared, slides were deparaffinised and stained with AntiOlfactory Marker Protein antibodies (Sigma, Dorset, UK).

Through a process of trial and error we found that antibodies at a dilution of 1:300 were optimal, and that no pre-treatment of slides was necessary. Assays were performed on the Bond Max automated immuno-stainer using the Bond Polymer Refine detection system (following manufacturer's instructions) using staining protocol $\mathrm{F}$ : peroxide block $5 \mathrm{~min}$, primary $15 \mathrm{~min}$, post primary $8 \mathrm{~min}$, polymer $8 \mathrm{~min}$, DAB $10 \mathrm{~min}$, haematoxylin $5 \mathrm{~min}$.

\section{Testing of nasal polyps}

Subsequently consecutive adult patients undergoing surgical nasal polypectomy at Kettering General Hospital between November 2007 and May 2009 were invited to participate in the study. Only those patients found (intra-operatively) to have polyps medial to the middle turbinate were recruited to the study. All macroscopic polyps found juxtaposed to 
olfactory epithelium were excised for analysis: from their base and without maceration. Excised tissue was processed for light microscopy and immunohistochemistry as described above. We also classified the extent of disease in these cases based upon radiological criteria. We classified the overall severity of sinonasal disease using the Lund-Mackay score ${ }^{(5)}$, and also rated the degree of soft tissue surrounding the olfactory cleft on each side. For the latter we followed the method of Konstantinidis et al., () for Damm nasal segment B3 ${ }^{(7)}$ : score 0 for no abnormality, 1 for partial opacification, and 2 for total opacification.

Ethical approval for this study was granted by Leicestershire, Northamptonshire, and Rutland Research Ethics Committee.

\section{RESULTS}

Using this protocol, we were able to demonstrate staining in the lamina propria of our sample of normal olfactory epithelium (Figures 1 and 2). There was no staining elsewhere, and the pattern and location of staining is consistent with specific staining of olfactory structures.

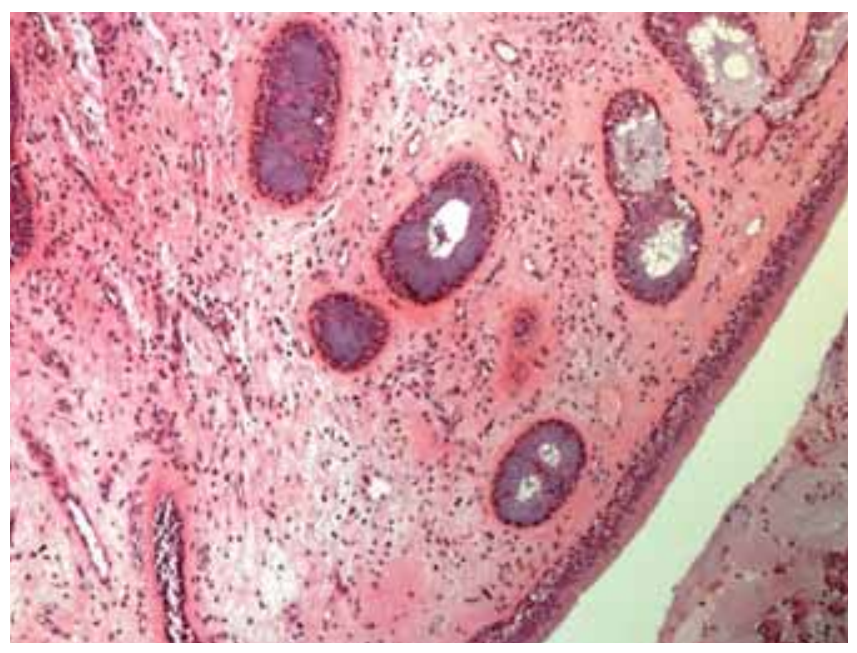

Figure 1. Cadaveric olfactory mucosa sample: H\&E stain (x400).

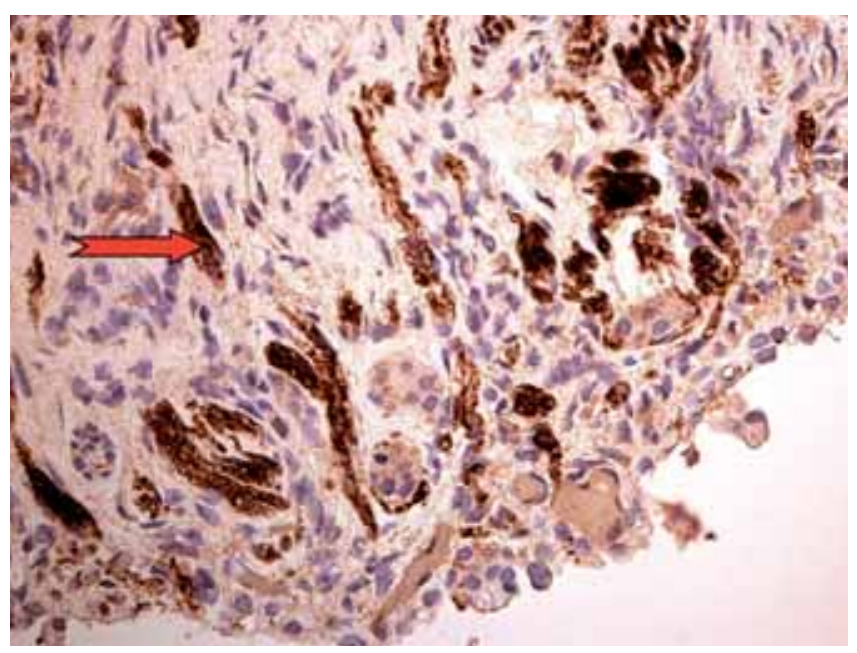

Figure 2. Cadaveric olfactory mucosa sample: anti-OMP staining (example of staining highlighted with arrow) (x200).
In the study period, 12 patients with nasal polyposis were eligible to participate, and a total of 25 surgical polypectomy samples from these patients were sent for histological analysis. These samples were derived from a variety of anatomical regions juxtaposed to areas known to contain olfactory epithelium ${ }^{(8)}$ and were of a varied size (Table 1). The LundMackay score of participants varied from 13 to 22, with a mean score of 16.1. All participants showed radiological evidence of opacification in the region of the olfactory cleft. Five $(42 \%)$ of the participants had bilateral total opacification, but six $(20 \%)$ showed only partial opacification, at least on one side. One participant did not have radiological imaging prior to surgery.

All of the 25 samples in the study showed histological features typical of inflammatory nasal polyps ${ }^{(9)}$ : an eosinophilrich inflammatory mass lined by respiratory epithelium with occasional areas of squamous metaplasia. No polyps demonstrated staining with anti-OMP in any anatomical region (Figures 3 and 4). This provides good evidence for the absence of olfactory structures in nasal polyps, but with a

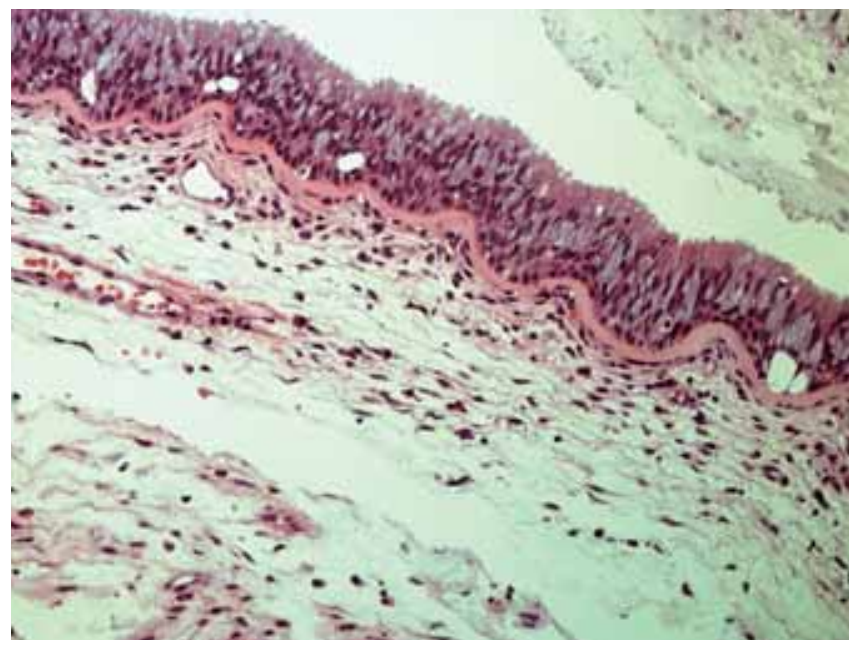

Figure 3. Nasal polyp arising from the anterior superior turbinate: H\&E stain $(x 400)$.

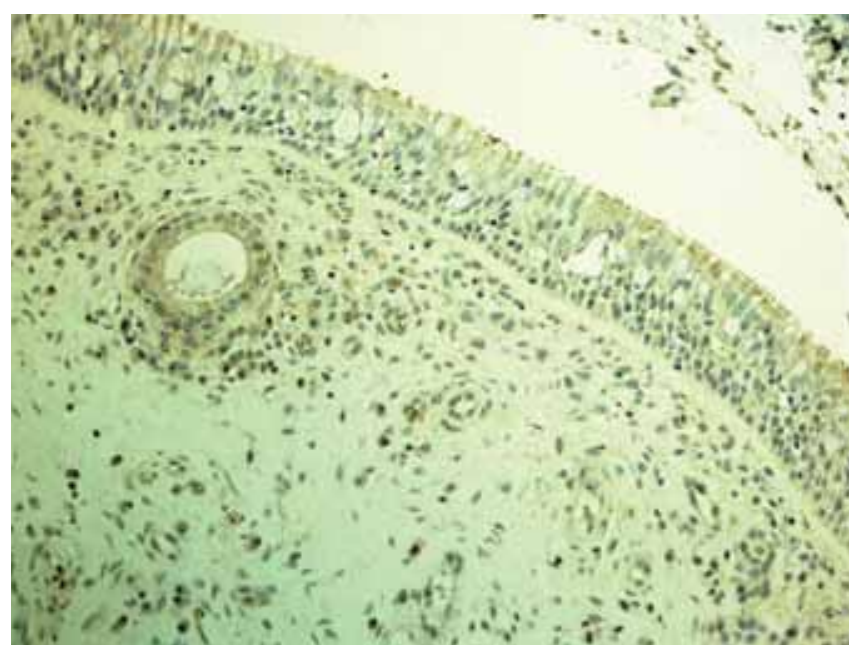

Figure 4. Nasal polyp arising from the anterior superior turbinate: no staining with anti-OMP (x200). 


\begin{tabular}{|c|c|c|c|c|c|c|c|c|c|}
\hline \multirow[b]{3}{*}{ Participant } & & \multicolumn{5}{|c|}{ Anatomical Site \& Size $(\mathrm{mm})$ of Excised Polyps } & \multicolumn{3}{|c|}{ Radiological Scoring } \\
\hline & \multirow[b]{2}{*}{ Age } & \multicolumn{2}{|c|}{$\begin{array}{c}\text { Anterior } \\
\text { superior turbinate }\end{array}$} & \multicolumn{2}{|c|}{$\begin{array}{c}\text { Posterior } \\
\text { superior turbinate }\end{array}$} & \multirow[t]{2}{*}{$\begin{array}{l}\text { Antero-superior } \\
\text { septum }\end{array}$} & \multirow{2}{*}{$\begin{array}{c}\text { Lund-Mackay } \\
\text { Score }\end{array}$} & \multicolumn{2}{|c|}{ Olfactory Cleft score } \\
\hline & & Right & Left & Right & Left & & & Right & Left \\
\hline 1 & 67 & & $18 \times 8$ & & $\begin{array}{c}25 \times 13 \\
12 \times 6\end{array}$ & & 16 & 2 & 2 \\
\hline 2 & 56 & $10 \times 10$ & & & $26 \times 8$ & & 14 & 1 & 1 \\
\hline 3 & 46 & $22 \times 15$ & $15 \times 8$ & $20 \times 16$ & $19 \times 15$ & & 22 & 2 & 2 \\
\hline 4 & 62 & $27 \times 20$ & $35 \times 30$ & & & & 14 & 1 & 1 \\
\hline 5 & 63 & & $9 \times 6$ & & $10 \times 6$ & & 15 & 2 & 2 \\
\hline 6 & 53 & & $20 \times 8$ & & $20 \times 9$ & & 17 & 1 & 2 \\
\hline 7 & 61 & $13 \times 8$ & & & & & 20 & 2 & 2 \\
\hline 8 & 60 & & $5 \times 4$ & & & & 13 & 1 & 1 \\
\hline 9 & 62 & & & $20 \times 10$ & $25 \times 20$ & & 16 & 1 & 1 \\
\hline 10 & 62 & & $20 \times 19$ & & & $10 \times 10$ & 16 & 1 & 1 \\
\hline 11 & 69 & $8 \times 8$ & & $22 \times 18$ & $15 \times 15$ & & & Unknown & \\
\hline 12 & 64 & & $12 \times 10$ & & & & 14 & 1 & 1 \\
\hline
\end{tabular}

Table 1. Anatomical origin and size of nasal polyps analysed in this study, and radiological grading of severity of disease. For details see text.

binomial 95\% confidence interval of $0-13.7 \%$.

\section{DISCUSSION}

Nasal polyps are oedematous inflammatory masses arising from the mucosa of the lateral nasal wall, and form part of the spectrum of chronic rhinosinusitis ${ }^{(10)}$. Their cause is uncertain, but they probably represent a non-IgE mediated allergic type reaction of the nasal mucous membrane ${ }^{(11)}$. They can cause a variety of nasal symptoms, but the predominant complaint is usually one of nasal blockage. A significant number of patients also suffer from a poor sense of smell ${ }^{(12,13)}$. Olfactory dysfunction has a significant impact upon quality of life ${ }^{(14)}$, and so is an important symptom to treat, although it may be difficult to do so.

The cause of hyposmia in nasal polyposis is uncertain, but is likely to be multifactorial ${ }^{(15)}$. One factor is thought to be that inflammatory polyps cause a physical obstruction to odourants reaching the olfactory cleft (conductive loss). Polyps arising lateral to the middle turbinate are usually responsible for nasal obstruction, whereas those medial to this structure are associated with olfactory dysfunction ${ }^{(6)}$. Previous studies ${ }^{(6)}$ have suggested that the Lund-Mackay score, which is a radiological assessment of the overall severity of sinonasal pathology, does not positively correlate with measurable olfactory dysfunction. However, radiological evidence of thickening specifically in the olfactory region does positively correlate with subjective and objective hyposmia in such patients ${ }^{(6)}$, although this still may not be able to predict operative outcomes.

An alternative, although not mutually exclusive, cause for hyposmia in nasal polyposis may be physiological dysfunction of the olfactory neuroepithelium. A recent report ${ }^{(3)}$ of patients with nasal polyposis reported that the mucosa surrounding the olfactory neuroepithelium in such patients is often also inflamed (suggesting that the inflammatory process is generalized rather than discontinuous). The degree of inflammation in this study correlated to the extent of olfactory dysfunction.

In our study, we found that radiological total opacification of the olfactory cleft was present in many but not all cases. This supports the hypothesis that, in at least a subset of patients, hyposmia in nasal polyposis is due to local inflammation of the olfactory neuroepithelium, rather than a pure conductive loss.

The treatment of nasal polyps can be medical or surgical. Topical steroids can reduce the size of polyps and alleviate blockage, but they have little effect upon the sense of smell ${ }^{(16)}$. Systemic steroids may also be used, and these may help with the sense of smell as well as with the symptoms of blockage (16,17). Surgery is used for disease resistant to medical therapy, and may create improvements in olfactory sensation ${ }^{(18-23)}$, but the improvement is seen only in some cases and is not always sustained ${ }^{(18,24)}$. The effect of surgery on olfactory function in nasal polyposis may be misrepresented, because many studies used subjective rather than objective measures of smell ${ }^{(25)}$, even though many objective tests of olfaction are available ${ }^{(26,27)}$.

Surgical strategy for polyps medial to the middle turbinate is an area of controversy. Isolated polyps in this area should probably be investigated further, because they may represent something other than simple nasal polyps, such as respiratory adenomatoid hamartoma ${ }^{(28,29)}$ or an encephalocoele ${ }^{(30)}$. However, when such polyps are present as part of a more diffuse polyposis, they are likely to represent simple inflammatory polyps. Many advocate excision of such polyps, to reduce any conductive obstruction to olfaction. Others suggest that these polyps should not be excised (31), because of the risk that the polyps themselves, or the area surrounding them, may contain olfactory neuroepithelium ${ }^{(32)}$, 
with potential permanently injury to olfactory sensation from surgical trauma. This risk may be compounded by difficult access to excision of polyps from this region ${ }^{(33)}$.

It is known that in general nasal polyps are insensate suggesting no sensory innervation, but neural elements have occasionally been found in the base of polyps ${ }^{(34,35)}$. In addition, olfactory neurons do have unusual capacities for regeneration and growth ${ }^{(36)}$. Studies of nasal mucosa in participants without polyps have shown that areas of olfactory and respiratory epithelium can become intermingled ${ }^{(37)}$. Thus, there are many reasons to suggest that nasal polyps could contain olfactory structures. To date no study has looked to specifically refute this possibility.

In this study, using immunohistochemical staining for olfactory neuronal components, we have shown that nasal polyps do not (or at least rarely) contain olfactory components.

Our study has some limitations. Although we stained for antiOMP, we did not use antibody against markers that have been used in other studies, such as PGP 9.5 or beta-tubulin ${ }^{(3)}$, but the positive staining in a normal specimen of olfactory mucosa using our protocol suggests that our results are valid. Our study is small, and so although none of the 25 polypectomy specimens here showed olfactory components, the upper end of the $95 \%$ confidence interval is $13.7 \%$. Finally, we did not measure olfactory outcomes from our study group following polypectomy: because that was not the aim of our study, and because in any case the effect size would likely be too small for our small sample size to show.

We cannot make a general recommendation or risk/benefit analysis as to whether polyps medial to the middle turbinate should be removed in an attempt to improve hyposmia from our study. Given the probable multifactorial aetiology of hyposmia in nasal polyposis, the decision to surgically excise polyps in the olfactory region is likely to be an individual patient decision, but based upon prognostic indicators that (at present) we cannot reliably predict ${ }^{(38)}$. However, we have shown here that there is no absolute contraindication to the careful removal of polyps surrounding the olfactory cleft, because they do not (or at least rarely do) contain olfactory components.

\section{ACKNOWLEDGEMENT}

This study was supported by a grant from the Kettering General Hospital ENT Research Fund.

\section{AUTHOR CONTRIBUTION: MB, SA and JU conceptualized} the research project. $\mathrm{MB}, \mathrm{RL}$ and $\mathrm{ML}$ wrote the paper.

The authors declare no conflict of interest.

\section{REFERENCES}

1. St John JA, Key B. Olfactory marker protein modulates primary olfactory axon overshooting in the olfactory bulb. J Comp Neurol.
2005; 488: 61-69.

2. Say P, Leopold D, Cochran G, Smith L, Greiner T. Resection of the inferior superior turbinate: does it affect olfactory ability or contain olfactory neuronal tissue? Am J Rhinol 2004; 18: 157-160.

3. Konstantinidis I, Witt M, Kaidoglu K, Constantinidis J, Gudziol V. Olfactory mucosa in nasal polyposis: Implications for FESS outcome. Rhinology. 2010; 48: 47-53.

4. Nakashima T, Kimmelman CP, Snow JB Jr. Immunohistopathology of human olfactory epithelium, nerve and bulb. Laryngoscope. 1985; 95: 391-396.

5. Lund VJ, Kennedy DW. Staging for rhinosinusitis. Otolaryngol Head Neck Surg. 1997; 117: S35-40.

6. Konstantinidis I, Triaridis S, Printza A, Vital V, Ferekidis E, Constantinidis J. Olfactory dysfunction in nasal polyposis: correlation with computed tomography findings. ORL; journal for oto-rhino-laryngology and its related specialties 2007; 69: 226-232.

7. Damm M, Vent J, Schmidt M, et al. Intranasal volume and olfactory function. Chem Senses. 2002; 27: 831-839.

8. Leopold DA, Hummel T, Schwob JE, Hong SC, Knecht M, Kobal G. Anterior distribution of human olfactory epithelium. Laryngoscope. 2000; 110: 417-421.

9. Michaels L, Hellquist HB. Ear, Nose and Throat Histopathology. London: Springer-Verlag, 2001.

10. Fokkens W, Lund V, Mullol J. European position paper on rhinosinusitis and nasal polyps 2007. Rhinol Suppl. 2007: 1-136.

11. Mygind N, Dahl R, Bachert C. Nasal polyposis, eosinophil dominated inflammation, and allergy. Thorax. 2000; 55 Suppl 2: S79-83.

12. Vento SI, Simola M, Ertama LO, Malmberg CH. Sense of smell in long-standing nasal polyposis. Am J Rhinol. 2001; 15: 159-163.

13. Litvack JR, Fong K, Mace J, James KE, Smith TL. Predictors of olfactory dysfunction in patients with chronic rhinosinusitis. Laryngoscope. 2008; 118: 2225-2230.

14. Nordin S, Bramerson A. Complaints of olfactory disorders: epidemiology, assessment and clinical implications. Curr Opin Allergy Clin Immunol. 2008; 8: 10-15.

15. Raviv JR, Kern RC. Chronic sinusitis and olfactory dysfunction. Otolaryngol Clin North Am. 2004; 37: 1143-1157, v-vi.

16. Mygind N, Lund V. Intranasal corticosteroids for nasal polyposis: biological rationale, efficacy, and safety. Treat Respir Med. 2006; 5: $93-102$

17. Jafek BW, Moran DT, Eller PM, Rowley JC, 3rd, Jafek TB. Steroid-dependent anosmia. Arch Otolaryngol Head Neck Surg. 1987; 113: 547-549.

18. Klimek L, Moll B, Amedee RG, Mann WJ. Olfactory function after microscopic endonasal surgery in patients with nasal polyps. Am J Rhinol. 1997; 11: 251-255.

19. Jankowski R, Bodino C. Olfaction in patients with nasal polyposis: effects of systemic steroids and radical ethmoidectomy with middle turbinate resection (nasalization). Rhinology. 2003; 41: 220-230.

20. Litvack JR, Mace J, Smith TL. Does olfactory function improve after endoscopic sinus surgery? Otolaryngol Head Neck Surg. 2009; 140: 312-319.

21. Perry BF, Kountakis SE. Subjective improvement of olfactory function after endoscopic sinus surgery for chronic rhinosinusitis. Am J Otolaryngol. 2003; 24: 366-369.

22. Olsson P, Stjarne P. Endoscopic sinus surgery improves olfaction in nasal polyposis, a multi-center study. Rhinology; 48: 150-155.

23. Bonfils P. Evaluation of the combined medical and surgical treatment in nasal polyposis. I: functional results. Acta OtoLaryngologica. 2007; 127: 436-446.

24. Blomqvist EH, Lundblad L, Anggard A, Haraldsson PO, Stjarne P. A randomized controlled study evaluating medical treatment versus surgical treatment in addition to medical treatment of nasal polyposis. J Allergy Clin Immunology. 2001; 107: 224-228.

25. Bonfils P, Malinvaud D, Soudry Y, Devars du Maine M, Laccourreye O. Surgical therapy and olfactory function. B-ENT. 2009; 5 Suppl 13: 77-87.

26. Rombaux P, Collet $\mathrm{S}$, Martinage $\mathrm{S}$, et al. Olfactory testing in clinical practice. B-ENT. 2009; 5 Suppl 13: 39-51.

27. Simmen D, Briner HR. Olfaction in rhinology--methods of 
assessing the sense of smell. Rhinology. 2006; 44: 98-101.

28. Cao Z, Gu Z, Yang J, Jin M. Respiratory epithelial adenomatoid hamartoma of bilateral olfactory clefts associated with nasal polyposis: three cases report and literature review. Auris, nasus, larynx; 37: 352-356.

29. Lima NB, Jankowski R, Georgel T, Grignon B, Guillemin F, Vignaud JM. Respiratory adenomatoid hamartoma must be suspected on CT-scan enlargement of the olfactory clefts. Rhinology. 2006; 44: 264-269.

30. Hoxworth JM, Glastonbury CM, Fischbein NJ, Dillon WP. Focal opacification of the olfactory recess on sinus CT: just an incidental finding? AJNR Am J Neuroradiol. 2008; 29: 895-897.

31. Simmen D, Jones, N. Manual of Endoscopic Sinus Surgery and Its Extended Applications. London: Thieme, 2005.

32. Kimmelman CP. The risk to olfaction from nasal surgery. Laryngoscope. 1994; 104: 981-988.

33. Masaki M, Tanaka Y. Nasal polyps in the olfactory cleft. Laryngoscope. 1998; 108: 1243-1246.

34. Sasaki Y, Nakahara H. Innervation of human nasal polyps. Rhinology. 1985; 23: 195-199.

35. Hiraide F, Kakoi H. Histochemical study on innervation of glands and blood vessels in nasal polyps. Acta Otolaryngol Suppl. 1986; 430: 5-11.
36. Margolis FL, Verhaagen J, Biffo S, Huang FL, Grillo M. Regulation of gene expression in the olfactory neuroepithelium: a neurogenetic matrix. Prog Brain Res. 1991; 89: 97-122.

37. Morrison EE, Costanzo RM. Morphology of the human olfactory epithelium. J Comp Neurol. 1990; 297: 1-13.

38. Seiden AM, Duncan HJ. The diagnosis of a conductive olfactory loss. Laryngoscope. 2001; 111: 9-14.

Mahmood Bhutta

Nuffield Department of Surgical Sciences (University of Oxford)

Level 6, John Radcliffe Hospital

Headley Way, Oxford OX3 9DU United Kingdom

Tel: +44-(0)1865-220532

Fax: +44- (0)1865-768876

E-mail:m.bhutta@doctors.org.uk 